\title{
Ciudad e identidad cultural. ¿Cómo se relacionan con lo urbano los indígenas amazónicos peruanos en el siglo XXI?
}

Ville et identité culturelle. Comment les indigènes de l'Amazonie péruvienne sont-ils reliés au facteur urbain au XXI ${ }^{e}$ siècle?

City and cultural identity. How are the amazon peruvian natives related with urban in the XXI century?

\section{Oscar Espinosa de Rivero}

\section{OpenEdition}

Journals

Edición electrónica

URL: http://journals.openedition.org/bifea/2799

DOI: 10.4000/bifea.2799

ISSN: 2076-5827

Editor

Institut Français d'Études Andines

Edición impresa

Fecha de publicación: 1 abril 2009

Paginación: 47-59

ISSN: 0303-7495

Referencia electrónica

Oscar Espinosa de Rivero, « Ciudad e identidad cultural. ¿Cómo se relacionan con lo urbano los indígenas amazónicos peruanos en el siglo XXI? », Bulletin de l'Institut français d'études andines [En línea], 38 (1) | 2009, Publicado el 01 octubre 2009, consultado el 10 diciembre 2020. URL : http:// journals.openedition.org/bifea/2799; DOI : https://doi.org/10.4000/bifea.2799

\section{(c) $($ () $\odot$}

Les contenus du Bulletin de l'Institut français d'études andines sont mis à disposition selon les termes de la licence Creative Commons Attribution - Pas d'Utilisation Commerciale - Pas de Modification 4.0 International. 
Bulletin de I'Institut Français d'Études Andines / 2009, 38 (1): 47-59

\title{
Ciudad e identidad cultural. ¿Cómo se relacionan con lo urbano los indígenas amazónicos peruanos en el siglo XXI*?
}

\author{
Oscar Espinosa de Rivero**
}

\begin{abstract}
Resumen
En las últimas décadas el número de indígenas amazónicos que vivien en ciudades ha incrementado de manera exponencial. Este nuevo fenómeno implica la revisión y modificación de prácticas ancestrales y obliga a interrogarse sobre el porvenir de las sociedades indígenas. En el presente artículo se presentan algunos avances de un proyecto de investigación en curso sobre los indígenas amazónicos del Perú y su relación con las ciudades y lo urbano. Se discute la forma en que la antropología sobre la amazonía ha abordado el tema de la modernización y urbanización en relación a la identidad cultural de los pueblos indígenas. También se presentan algunos resultados preliminares sobre el caso de las ciudades de Iquitos y Puerto Maldonado, así como la experiencia de los pueblos shipibo-konibo y asháninka con lo urbano. Finalmente se destaca el rol de la juventud indígena como factor decisivo en el futuro de los pueblos amazónicos.
\end{abstract}

Palabras clave: Amazonía, Perú, ciudad, urbano, Iquitos, Puerto Maldonado, shipibo, konibo, asháninka, juventud, identidad cultural

\footnotetext{
Una versión preliminar de este artículo fue presentada en la mesa redonda «Perspectivas en Antropología Amazónica» realizada el 17 de septiembre de 2008, como parte de las actividades de celebración de los 60 años del IFEA. Los resultados aquí presentados corresponden a los resultados preliminares de un proyecto de investigación más extenso sobre algunos de los desafíos que enfrentan los indígenas amazónicos en el siglo XXI. Parte de la investigación de campo en las ciudades amazónicas, en los años 2006 y 2007, fue financiada gracias a una beca del Legs Lelong del CNRS de Francia. Quisiera agradecer especialmente a Bonnie y Jean-Pierre Chaumeil por su confianza y apoyo. También quiero agradecer los interesantes y pertinentes comentarios de Roberto Pineda e Irène Bellier que me han permitido mejorar este texto.

** Coordinador de la especialidad de Antropología y Profesor Auxiliar del departamento de Ciencias Sociales de la Pontificia Universidad Católica del Perú. E-mail: oespinosa@pucp.edu.pe
} 


\title{
Ville et identité culturelle. Comment les indigènes de l'Amazonie péruvienne sont-ils reliés au facteur urbain au $\mathrm{XXI}^{\mathrm{e}}$ siècle?
}

\begin{abstract}
Résumé
Au cours des dernières décennies, un grand nombre d'Indiens originaires d'Amazonie s'en sont allés vivre en ville. Ce phénomène récent implique une révision et une modification des pratiques ancestrales et conduit à s'intérroger sur le devenir des sociétés indigènes. Dans cet article l'auteur présente les résultats préliminaires d'une enquête en cours sur les peuples indigènes de l'Amazonie péruvienne dans leurs relations avec les villes et le domaine urbain. L'article s'intéresse tout d'abord à la façon dont l'anthropologie amazonienne aborde le thème de la modernisation et de l'urbanisation dans ses relations à l'identité culturelle des peuples indigènes. L'auteur présente ensuite les résultats préliminaires de l'enquête menée à lquitos et Puerto Maldonado ainsi que l'expérience des peuples Shipibo-Konibo et Asháninka en milieu urbain. Finalement, le texte analyse l'importance du rôle joué par la jeunesse indigène dans l'évolution de ces populations.
\end{abstract}

Mot clés : Amazonie, Pérou, ville, urbain, Iquitos, Puerto Maldonado, shipibo, konibo, asháninka, jeunesse, identité culturelle

\section{City and cultural identity. How are the amazon peruvian natives related with urban in the XXI century?}

\begin{abstract}
In recent decades the number of indigenous Amazonian people living in cities has grown exponentially. This new phenomenon implies the revision and modification of ancestral practices and brings with it the immediate consequence of the question about the source of indigenous societies. In the present article some of the progress is presented of an ongoing project of investigation on indigenous Amazonian peoples of Peru and their relation with the cities and urban life. The form in which anthropology on the Amazon has dealt with the theme of modernization and urbanization in relation to cultural identity of indigenous communities is discussed. In addition, some of the preliminary results on the case of cities of Iquitos and Puerto Maldonado are presented, as is the urban experience of the Shipibo-Conibo and Ashninka. Finally, the role of the indigenous youth is highlighted as the decisive factor in determining the future of the Amazonian peoples of the 21st century.
\end{abstract}

Key words: Amazonia, Peru, city, urban, Iquitos, Puerto Maldonado, shipibo, konibo, asháninka, youth, cultural identity

La relación de los pueblos indígenas con las ciudades se remonta a muchos siglos atrás. Ya en tiempos precolombinos existían asentamientos humanos de grandes dimensiones en la región amazónica (Santos Granero, 1992). En el primer viaje hecho por europeos a través del río Amazonas, el cronista dominico, Gaspar de Carvajal (2002 [1542]), describía grandes asentamientos humanos en las riberas de este imponente río. Unos años después, otro cronista y conquistador español, Juan de Salinas Loyola, confirmaba la existencia de grandes ciudades a lo largo del río Ucayali (Salinas Loyola, 1965 [1557]; Myers, 1974). 
En las últimas décadas, el número de indígenas amazónicos viviendo en zonas urbanas de la Amazonía ha crecido exponencialmente, de tal manera que un porcentaje importante habita hoy en las diversas ciudades de la cuenca amazónica1. En el caso peruano, podemos encontrar a indígenas amazónicos que viven, no solamente en las grandes ciudades y capitales regionales como lquitos, Pucallpa o Puerto Maldonado, sino también en ciudades intermedias como La Merced, Satipo, Tingo María o Jaén; así como en pequeñas ciudades cuya población viene creciendo aceleradamente como Nauta, Nieva, Sepahua, San Lorenzo. Esta nueva situación plantea importantes interrogantes sobre las diferentes formas en que las sociedades indígenas se relacionan con lo urbano, sobre los desafíos que estas nuevas experiencias presentan a la vida política y las organizaciones indígenas y finalmente, sobre la supervivencia misma de las culturas indígenas en el futuro próximo.

\section{MODERNIDAD URBANA E IDENTIDAD CULTURAL}

Desde sus inicios, la antropología como disciplina ha mostrado una preocupación especial por el futuro de las culturas indígenas frente al impacto de la sociedad europea moderna. A principios del siglo XX, muchos antropólogos buscaban recolectar la mayor cantidad posible de información y de objetos de la cultura material indígena frente al temor de que pudieran desaparecer irremediablemente en el contexto del proceso de modernización acelerado que se vivía en el mundo entero. Estos objetos y materiales luego eran llevados a los museos para ser conservados y para ilustrar a la sociedad moderna cómo se vivía en otras épocas y lugares. A esta forma de acercarmiento a las culturas y sociedades indígenas se le ha denominado «antropología de salvataje»2.

Esta forma de proceder ha sido calificada recientemente por la antropóloga Mariza Peirano (2008) como uno de los «pecados» propios de la antropología. Según Peirano, esta manera de entender la antropología cosifica a la gente y sus culturas de tal modo que se llega a considerar que los indígenas han quedado atrapados en el pasado. Esta misma actitud que cosifica a las culturas indígenas y las congela en el pasado se ha difundido en otros sectores de la sociedad, sobre todo en los medios de comunicación y en el ámbito del turismo. Resulta particularmente ilustrativa la imagen que el gobierno peruano propaga a través de los videos producidos por PromPerú3, la agencia estatal que promueve el turismo. Uno de estos videos — que circuló ampliamente por casi dos años- mostraba imágenes de comunidades indígenas amazónicas mientras se escuchaba al narrador decir algo así como: «si quiere conocer el pasado...».

Hacia mediados del siglo XX, esta preocupación continuaba influenciando la reflexión de la antropología sobre las poblaciones indígenas. Claude Lévi-Strauss (1973) relata con nostalgia cómo el mundo indígena desaparece aceleradamente. Esta nostalgia se puede percibir en uno de sus libros más importantes: «Tristes Trópicos»4. Unas décadas después, el

1 Una de las principales dificultades para esta investigación es que no existen estadísticas sobre la población indígena viviendo en las ciudades. Los censos de 1993 y 2007 solo han censado a la población indígena amazónica que vive en las comunidades rurales, no en las ciudades, y aún así no han podido llegar a todas las comunidades existentes. La única información aproximada podría provenir de la pregunta por la lengua materna. Sin embargo, un porcentaje importante de la población indígena oculta a los encuestadores que su lengua materna es indígena por temor a ser discriminados.

2 También conocida como salvage anthropology en inglés o antropologia de salvamento en portugués.

3 Los videos de PromPerú aquí mencionados estuvieron disponibles en la página web de esta institución durante algún tiempo. Eventualmente se podrían solicitar a dicha institución.

4 Más revelador es el título que lleva en la edición inglesa: A World on the Wane («un mundo que se desvanece»). 
antropólogo brasileño, Darcy Ribeiro, expresaba también en su obra el riesgo de la posible desintegración étnica de los indígenas amazónicos. Según Ribeiro:

«las relaciones de la sociedad nacional con las tribus indígenas se procesan como un enfrentamiento entre entidades étnicas mutuamente exclusivas. Dada la desproporción demográfica y de nivel evolutivo que existe entre ellas, la interacción representa una amenaza permanente de desintegración de las etnias tribales» (1973).

Ribeiro quería mostrar cómo los rasgos étnicos más propios de cada pueblo indígena desaparecían debido al proceso de aculturación que estas sociedades venían enfrentando, y cómo daban lugar entonces a la aparición de lo que la antropología brasileña ha postulado como un «indio genérico» o «destribalizado» (Galvão, 1979; Ribeiro, 1986). A este proceso de aculturación, Ribeiro (1973) lo ha denominando «transfiguración étnica», y correspondería a lo que el antropólogo peruano Carlos Mora (1995) considera el proceso de «cholificación» en el caso de la Amazonía peruana.

La imagen de un indio «genérico» también aparece graficada en otro video más reciente de PromPerú5. En este video promocional, de muy corta duración, se ve a un hombre con el torso desnudo navegando por un río amazónico en una pequeña canoa. Lo único que se escucha es el ruido del agua y de los animales del bosque. Este hombre puede ser un indígena o no, porque a diferencia de las fotos de indígenas que aparecen en otras promociones turísticas, no posee rasgos que lo puedan identificar claramente con algún pueblo en particular: no usa kushma —ni de color pardo como los asháninka o mastsiguenga, ni de color blanco y con diseños geométricos como los shipibo o yine-, no lleva una corona hecha de plumas de tucán como los awajún o achuar, y tampoco se viste con «champas» de fibras vegetales como los yagua. Es decir, no presenta rasgos culturales definidos que lo identifiquen con un pueblo en particular. Este podría ser un ejemplo de cómo es imaginado el indio «genérico» o «destribalizado» desde el Estado o desde la ideología hegemónica de nuestra sociedad.

A principios del siglo XXI, todavía es posible escuchar comentarios de distintas personas que muestran su preocupación sobre la supervivencia de los pueblos indígenas en el futuro. Los procesos de escolarización, urbanización y modernización conducirían a la desaparición paulatina de las lenguas y las costumbres propias de los pueblos indígenas amazónicos. Además, esta posibilidad de aculturación y de pérdida de las identidades indígenas se podría acelerar en el contexto de expansión de la globalización capitalista.

Un aspecto particularmente problemático para la identidad y forma de vida tradicional indígena se puede percibir a partir de los cambios producidos en la relación entre los indígenas y el bosque amazónico. Eduardo Viveiros de Castro (2004) nos recuerda cómo para las sociedades amazónicas es fundamental el vínculo que existe entre las personas y la naturaleza, mientras que las relaciones sociales son algo más inestables. Por ello, la desaparición de los bosques debido a la deforestación y a la sobreexplotación de recursos naturales, así como la migración de los indígenas hacia las ciudades atentaría directamente sobre esta dimensión fundamental de la cosmovisión y la organización social amazónicas.

Sin embargo, también es posible una visión más optimista acerca del futuro de los pueblos indígenas. Personalmente estoy convencido que los tiempos actuales también ofrecen nuevas posibilidades a los pueblos indígenas para continuar recreando sus tradiciones culturales y fortalecer su propia identidad (Espinosa, 1998; 2007a).

5 Puede encontrarse en: http://www.youtube.com/watch?v=0Ysv90xyplM 
Un primer dato importante para sustentar este optimismo se encuentra en el crecimiento demográfico. Durante las tres últimas décadas del siglo XX, la población indígena de toda la cuenca amazónica se triplicó, y hoy en día sigue creciendo de manera estable ${ }^{6}$.

Una segunda razón para afianzar nuestra confianza radica en la existencia de una actitud más positiva que favorece y valora la diversidad cultural. La crisis de la razón occidental y de la visión lineal del progreso, típica de la posmodernidad, ha permitido revalorizar el aporte de las culturas indígenas. En este sentido, existe una mayor sensibilidad en la población en general, en el mundo entero, y sobre todo en la juventud, para valorar y respetar las diferencias culturales.

Una tercera señal positiva la encontramos en el reconocimiento formal de parte de los Estados y del sistema de Naciones Unidas de los derechos colectivos que poseen los pueblos indígenas. En septiembre del año 2007, la Asamblea General de las Naciones Unidas aprobó la Declaración sobre los Derechos de los Pueblos Indígenas, consolidando así una tendencia ya iniciada con el Convenio 169 de la Organización Internacional del Trabajo (OIT) para el establecimiento de mecanismos legales que protejan los derechos de los pueblos indígenas.

Evidentemente, estas señales positivas no anulan la existencia de conflictos y de problemas que tienen que enfrentar los indígenas amazónicos. Hoy en día muchos pueblos indígenas se encuentran en situaciones de gran vulnerabilidad debido a la reducción de sus territorios y recursos naturales, a problemas de salud, o sufren discriminación, marginación y violencia. Para evitar ser discriminados, muchos jóvenes indígenas optan por ocultar los signos externos de su identidad indígena, dejando de hablar sus idiomas o reprimiendo la expresión pública de otros aspectos propios de su herencia cultural.

Finalmente, habría que añadir que la historia de los pueblos indígenas amazónicos nos demuestra el potencial que tienen para enfrentar desafíos que incluso ponen en riesgo su supervivencia física. No es la primera vez que han enfrentado situaciones conflictivas o violencias extremas. Por el contrario, en contextos histórico-sociales muy difíciles han logrado renovar, transformar y reinterpretar muchos de sus patrones culturales tradicionales.

Un caso que ilustra estas capacidades ha sido la manera en que el pueblo asháninka ha enfrentado la experiencia de la violencia política que vivió en las últimas décadas del siglo pasado. Se calcula que más de 6 mil asháninkas murieron, más de 5 mil estuvieron secuestrados por Sendero Luminoso, más de 10 mil fueron obligados a desplazarse a otras zonas y regiones, decenas de niños quedaron huérfanos, llegando a desaparecer entre 30 y 40 comunidades locales indígenas durante esos años. Sin embargo, el pueblo asháninka supo utilizar sus propios recursos culturales — su conocimiento del bosque, sus habilidades como guerreros, su creatividad — no solamente para sobrevivir, sino también para lidiar con circunstancias particularmente adversas como la tortura psicológica, el duelo frente a los familiares asesinados y la posterior convivencia comunitaria con personas que estuvieron en el bando contrario (Espinosa, 1994; Villapolo \& Vásquez, 1999; Comisión de la Verdad y Reconciliación, 2003; Villapolo, 2003; Barrantes, 2007).

En este sentido, los procesos de urbanización contemporáneos constituyen uno de los diversos desafíos que enfrentan los pueblos indígenas amazónicos hoy en día. A pesar de ello, el tema de la relación de los indígenas con las ciudades y lo urbano todavía no

6 En el caso peruano la población indígena amazónica sigue manteniendo un ritmo de crecimiento importante. Según los censos oficiales (pero incompletos) se ha pasado de 299218 indígenas amazónicos en el año 1993 a 332975 en el año 2007, lo que implica un crecimiento del 11 \%. Por supuesto, ambos censos no toman en cuenta a la población indígena viviendo en las ciudades. 
ha sido asumido con la seriedad que amerita: ni por los sectores académicos, ni por el Estado, ni tampoco por las organizaciones indígenas. En las páginas siguientes quiero presentar algunos resultados iniciales sobre el estudio preliminar que estoy realizando al respecto en el Perú. Espero que éstos puedan convertirse en pistas de investigación que animen a otras personas a pensar también sobre este tema crucial para el futuro de los pueblos amazónicos.

\section{DIFERENTES EXPERIENCIAS DE LO URBANO}

En primer lugar, no todos los pueblos indígenas de la Amazonía se relacionan de la misma manera con lo urbano. En algunos casos se prefiere migrar hacia las grandes ciudades. En general, esta migración se orienta principalmente hacia las ciudades que se encuentran en la misma región amazónica, ya que permiten a los migrantes no alejarse tanto del mundo social y natural del que provienen. A primera vista, pareciera ser que en el caso de otras ciudades peruanas no amazónicas la migración ha sido menor, aunque aparentemente la migración hacia Lima se vendría incrementando en los últimos años debido a la concentración de servicios y de ofertas de empleo.

En realidad, es difícil saber con exactitud cuántos indígenas amazónicos viven en las ciudades debido a la ausencia de información censal. El Estado peruano no ha mostrado interés en obtener dicha información en sus censos nacionales ni en ningún otro tipo de encuesta oficial que organiza el Instituto Nacional de Estadística e Informática (INEI). Incluso, en los dos únicos casos en que se ha censado a la población indígena peruana (1993 y 2007), los censos solo han tomado en cuenta a la población viviendo en comunidades rurales y no a los indígenas que viven en las ciudades. Por ello, es probable que la población indígena que vive en Lima o en otras ciudades del país sea estadísticamente interesante.

Eventualmente, en estas ciudades solo se llega a conocer la existencia de población indígena amazónica cuando se expresa de manera visible a través de manifestaciones públicas en las calles o en los medios de comunicación. Por supuesto, también es posible enterarse de manera informal a través de los datos que circulan entre los propios indígenas y sus redes sociales7.

A continuación nos referiremos a cuatro casos que hemos venido estudiando: dos de ellos corresponden a la experiencia de los indígenas en las ciudades amazónicas de lquitos y Puerto Maldonado; mientras que los otros dos casos corresponden a estrategias particulares de dos pueblos indígenas —el shipibo-konibo y el asháninka— frente a lo urbano.

\section{1. Los pueblos indígenas en la ciudad de Iquitos}

Iquitos es la ciudad históricamente más importante y con mayor población de la Amazonía peruana. Según el censo nacional del año 2007, la ciudad de lquitos tendría una población apromixamada de 380 mil habitantes. De este total, se calcula que entre el 60 y $80 \%$ es indígena o se considera descendientes de indígenas. Es decir, estamos hablando de una población indígena en Iquitos entre 220 y 300 mil habitantes, aproximadamente. Actualmente no se cuenta con información exacta sobre el número de indígenas que viven en la ciudad ni sobre su origen étnico. Ahora bien, a diferencia de Pucallpa y de las ciudades

7 Por ejemplo, solamente a través de estos medios informales he logrado conocer la existencia de una importante red de vigilantes indígenas yánesha que viven y trabajan en la ciudad de Lima. 
de la Selva Central, en Iquitos viven indígenas procedentes de distintas etnias de la región. La información que se maneja, de manera más intuitiva, nos indica que gran porcentaje de la población indígena que vive en lquitos pertenece a los pueblos boráa (bora), witoto (huitoto), yagua, tikuna, ocaina, y sobre todo kukama (cocama) y kichwa8.

La mayor parte de indígenas que viven de manera permanente en la ciudad de lquitos no expresa públicamente su identidad étnica de manera visible, aunque muchos sí lo hacen al interior de sus propias redes sociales o entre sus paisanos. Esto ocurre también en el caso de aquellos que se consideran a sí mismos como «descendientes» de indígenas. La explicación de este ocultamiento de la identidad indígena podría deberse a la discriminación vivida durante décadas y que ha generado un sentimiento de vergüenza que se ha arraigado profundamente entre la población de origen indígena, tal como ocurre en otras regiones del país.

Este no es, sin embargo, el caso de los dirigentes indígenas o de los jóvenes que se encuentran estudiando en la universidad o en institutos superiores. En general, éstos no tienen mayores dificultades en expresar públicamente su identidad como indígenas, sobre todo si se trata de hacerlo para reivindicar sus derechos. Es posible que esta actitud diferente se deba a que los dirigentes indígenas han aprendido que a los medios de comunicación les gusta mostrar el aspecto más «fotogénico» y «exótico» de los indígenas. En el contexto de una demanda política, para los indígenas amazónicos les resulta más redituable el vestirse con sus trajes tradicionales para atraer la atención de las autoridades y de los medios periodísticos.

Al mismo tiempo, la mayoría de peruanos, siguiendo la ideología hegemónica, restringe la identidad indígena solo a aquellas personas que viven en comunidades indígenas rurales. Por ello, en Iquitos se suele considerar que los indígenas que viven en la ciudad ya no son propiamente indígenas. Muchas veces estas ideas también son compartidas por los dirigentes y las organizaciones indígenas, de tal manera que no tienen una política especial para atender a los indígenas urbanos, que quedan así totalmente invisibilizados, incluso para otros indígenas como ellos.

\section{2. Los pueblos indígenas y la ciudad de Puerto Maldonado}

Puerto Maldonado es la capital de la región de Madre de Dios y cuenta con aproximadamente 56 mil habitantes según el censo del año 2007. En la región de Madre de Dios existen treinta y un comunidades nativas, que representan una población de cuatro mil indígenas, aproximadamente9. Estos indígenas pertenecen a los pueblos harakmbut, ese'eja, matsiguenka, yine y shipibo, y en menor medida a otros pueblos.

Para la mayor parte de indígenas de esta región, Puerto Maldonado constituye una ciudad «blanca»o «mestiza», es decir, «no indígena», ajena a sus propias culturas y tradiciones. Esta percepción es compartida incluso por aquellos indígenas que optan por vivir en la ciudad de manera más estable. Sin embargo, esto no altera la importancia que Puerto Maldonado tiene para los indígenas de la región en tanto centro político y comercial. Como toda ciudad grande, Puerto Maldonado constituye un lugar atractivo para los indígenas en la medida en que ofrece la posibilidad de satisfacer algunas de sus necesidades puntuales, como la realización de trámites oficiales, la posibilidad de continuar sus estudios y el intercambio

8 Esta información ha sido proporcionada por las organizaciones indígenas, funcionarios del Estado, promotores de ONG, periodistas, expertos en temas indígenas y pobladores indígenas de la ciudad de lquitos.

9 Según el censo INEI de 2007 serían 4005 indígenas. Sin embargo, es posible que algunas comunidades no hayan sido adecuadamente censadas, con lo cual el número podría incrementarse un poco más. 
comercial. Sin embargo, en tanto ciudad «blanca» o «mestiza», no es el mejor lugar para vivir. Es tan solo un lugar al que se recurre de manera constante, pero siempre por periodos de tiempo claramente definidos.

A pesar de ello, algunas personas o familias indígenas optan por migrar a la ciudad buscando los atractivos que la modernidad les ofrece. Según los propios indígenas que viven en Puerto Maldonado, la mayoría de los que optan por una migración de tipo permanente terminan invisibilizándose como indígenas. Esto se debería, según mis informantes, a que no existe compatibilidad entre la forma de vida indígena y la forma de vida urbana. Sin embargo, habría una excepción: la del pueblo shipibo. Efectivamente, para los otros pueblos indígenas de Madre de Dios, los shipibo son los únicos que mejor se han adaptado a la vida urbana debido al mayor tiempo de contacto que tienen con la sociedad «blanca» $\mathrm{o} \ll$ mestiza».

\section{3. El pueblo shipibo-konibo}

El caso de los shipibo-konibo es particularmente interesante, ya que se trataría del pueblo indígena de la Amazonía peruana con la mejor y más estable relación con el medio urbano moderno. Se calcula que entre el 18 y el 25 \% de la población shipibo-konibo actual estaría viviendo en el eje urbano Pucallpa-Yarinacocha (Tournon, 2002). Sin embargo, según el censo del año 2007 se calculó que los shipibo-konibo tan solo constituirían del 3 al $5 \%$ del total de la población de esta ciudad.

A pesar de ello, la presencia del pueblo shipibo en la ciudad de Pucallpa es bastante importante. Esto podría deberse al hecho de que se trata de una ciudad que ha sido fundada en medio de un territorio indígena, a diferencia de otras ciudades amazónicas, como Iquitos o Puerto Maldonado, que se han creado en zonas periféricas o intermedias entre territorios indígenas ancestrales.

Pero los shipibo no solo se han asentado en el eje urbano Pucallpa-Yarinacocha, sino que también viven en muchas otras ciudades importantes de la región como Tingo María, La Merced, Tarapoto, Yurimaguas, Puerto Maldonado, Iquitos y Lima ${ }^{10}$. En todos estos lugares, los shipibo se han organizado para garantizar su sustento, educar a sus hijos y defender sus derechos como indígenas.

Otro aspecto importante radica en que, a diferencia de otros pueblos indígenas, los shipibo-konibo expresan de manera pública y visible su condición como tales. Esto es especialmente cierto en el caso de las mujeres que, vestidas con sus trajes típicos, venden sus artesanías en lugares céntricos, sobre todo si es que hay afluencia de turistas. En algunas ciudades han establecido también campos feriales de artesanía que ellas administran, y un grupo en Lima está planificando construir una aldea tradicional shipiba donde puedan vivir a la usanza tradicional —ien medio del desierto y del hostil clima limeño! — y además convertirla en un destino turístico.

Parece ser que los pueblos que se dedican a la producción y comercio de artesanías suelen establecer una relación con la ciudad que les permite, por un lado, acceder a ciertos ingresos monetarios, y al mismo tiempo mantener o desarrollar espacios donde puedan expresar algunas de sus tradiciones culturales. En este sentido, el caso del pueblo shipibo no es único en la región amazónica, algo similar experimentan otros pueblos indígenas del río Negro que han migrado a la ciudad brasileña de Manaos (Jaramillo, 2003).

10 Probablemente también existan grupos organizados del pueblo shipibo en otras ciudades mestizas a lo largo del río Ucayali, como Contamana, Requena y Nauta, pero no tenemos información segura al respecto. 
El estudio de la historia y la vida política del pueblo shipibo-konibo (Espinosa, 2004) me lleva a creer, además, que es posible que diversas formas y patrones culturales indígenas tradicionales de los pueblos amazónicos no lleguen a desaparecer ante nuevos desafíos que aparecen a lo largo del tiempo, sino que por el contrario pueden transformarse y reinterpretarse en nuevos contextos histórico-sociales.

Esto puede apreciarse, por ejemplo, en el caso de nuevas versiones que circulan entre los shipibo-konibo de las historias tradicionales o mitos sobre la lupuna. La lupuna (Chorisia Insignis) es un árbol con un importante significado cosmogónico y cosmológico en las distintas culturas amazónicas11. En tiempos recientes, está circulando una versión que indica cómo algunos árboles de lupuna se resisten a la deforestación y a la tala depredadora de especies maderables en la región al adquirir una forma curva y enredada, y por lo tanto, menos valiosa para los madereros. En la zona urbana de Pucallpa-Yarinacocha existe, además, una gran plaza cuyo único árbol es una lupuna gigantesca. Este árbol y esta plaza constituyen un referente importante en la vida cotidiana y ritual de los shipibos que viven en la ciudad. Estos dos ejemplos sobre el árbol de lupuna y su lugar simbólico entre los shipibos urbanos muestran la pervivencia y transformación de un aspecto de la cultura tradicional indígena.

Además, los shipibos urbanos hacen su presencia visible a través de otras formas. Por una parte, son muy activos en la reivindicación de sus derechos indígenas. En Pucallpa han logrado establecer algunas fechas que celebran públicamente por las calles de la ciudad, con marchas, pasacalles y fiestas. Celebran, por ejemplo, el Día de los Pueblos Indígenas y el Día de la Educación Bilingüe Intercultural, además de participar en desfiles con ocasión de otras celebraciones propias de la región, como en el Día de la Primavera, en el Día del Maestro, o en Fiestas Patrias. En el caso de las ceremonias oficiales o eventos importantes de la ciudad, las autoridades o los dirigentes indígenas, como los consejeros regionales, regidores municipales y otros líderes, participan usando su traje de gala, que es la kushma shipiba.

Esta presencia pública también se refuerza a través del uso de los medios de comunicación modernos. Los shipibo cuentan con diversos programas radiales bilingües o en su propio idioma, así como cierta presencia en los diarios locales, e incluso existe un grupo de jóvenes muy animados que tienen un proyecto para crear un programa de televisión para las emisoras locales. Finalmente, habría que mencionar que existen grupos de familias shipibo que han comenzado a demandar escuelas bilingües para sus hijos en las ciudades. Hasta la fecha lo han solicitado en las ciudades de Pucallpa, Tingo María y también en Lima.

\section{4. El pueblo asháninka}

Finalmente vamos a presentar el caso del pueblo asháninka que habita en la Selva Central peruana. En esta región nos hemos centrado en las ciudades de La Merced y Satipo, que son capitales de las provincias de Chanchamayo y Satipo respectivamente. Al igual que en el caso de Pucallpa, se podría decir que estas dos ciudades se encuentran ubicadas en parte sobre el territorio ancestral asháninka. Sin embargo, esta zona ha sido intensamente colonizada desde una época muy temprana, sobre todo desde 1840, a diferencia de Pucallpa que comenzó a crecer recién un siglo después.

11 Existen numerosos mitos y leyendas en torno a la lupuna en distintas sociedades amazónicas además de la shipibokonibo, como la awajún, kukama, tikuna, yagua, etc. Al respecto existe una amplia bibliografía. Uno de los mitos más difundidos en el Perú es el de los yagua (Chaumeil, 1978). 
El proceso de colonización de la Selva Central ha sido tan intenso, que hoy en día las comunidades asháninka —al igual que las yánesha - constituyen, prácticamente, islas indígenas en un mar de asentamientos de colonos andinos. Hay que añadir, además, que este territorio también ha sido fuertemente depredado, habiendo desaparecido gran parte del bosque, dejando, por lo tanto, pocos espacios para la vida tradicional indígena. Frente a esta presión colonizadora, muchas familias asháninka optaron por la migración hacia territorios más lejanos e inaccesibles para los colonos mestizos. Así, durante la segunda mitad del siglo XX se puede apreciar un importante flujo migratorio desde el valle del río Perené hacia el nororiente, en dirección hacia el valle del río Pichis y del Ucayali, así como hacia el este, a los ríos Ene, Tambo, Urubamba y Purús.

En relación a su vínculo con lo urbano, el pueblo asháninka, a diferencia del pueblo shipibo, pero de manera similar a otros pueblos amazónicos como los tikuna en Brasil, han optado por llevar la ciudad a sus comunidades en vez de migrar a ésta. Es decir, están convirtiendo a algunas de sus comunidades más grandes en pequeñas «ciudades», de tal manera que les puedan permitir el acceso a diferentes bienes y servicios que se encuentran en la ciudad sin tener que emigrar. En algunos casos, la conversión de comunidades en pequeñas ciudades ha implicado también una reconfiguración urbanística al modificar el espacio físico de la comunidad, moviendo las casas y estableciendo calles y plazas.

\section{LA JUVENTUD INDÍGENA URBANA Y EL FUTURO DE LOS PUEBLOS AMAZÓNICOS}

Un último elemento importante a tener en cuenta, en este análisis preliminar, es el rol crucial que cumplen los jóvenes indígenas en el proceso de reconfiguración y adaptación de sus prácticas culturales, o en el abandono parcial o definitivo de éstas en el nuevo contexto urbano. Por lo tanto, se hace necesario comprender las diferencias generacionales que se están estableciendo, es decir, cómo los jóvenes indígenas se posicionan frente a la tradición de sus antepasados y al futuro de sus propios pueblos.

Cada vez hay más jóvenes indígenas que nacen y crecen en un mundo radicalmente diferente al de sus padres, muchos de ellos en contextos urbanos. La mayoría tienen acceso a la educación secundaria y un grupo creciente también a la educación superior. Al mismo tiempo, estos jóvenes indígenas están en contacto más directo con las nuevas tecnologías, sobre todo si viven en las ciudades y tienen acceso a Internet. Las nuevas tecnologías de la información y la comunicación les permiten entrar en contacto con gente de diversas culturas y de muchos países. Para algunos, estos contactos han significado la posibilidad de viajar a otras partes del mundo, a Europa, a Norteamérica. También jóvenes europeos y norteamericanos han venido a visitar sus comunidades y conocer su cultura.

Resulta particularmente importante el acceso a la educación superior, pues siempre se da en un contexto urbano y porque prepara profesionales para laborar en el mundo urbano moderno. Sin embargo, existen diversos desafíos que la juventud indígena tiene que enfrentar al respecto. Un primer desafío es el difícil acceso a la educación superior. A pesar de los cambios producidos en la sociedad peruana en las últimas décadas y de los frutos que han producido la lucha y actividad política de las organizaciones indígenas, el acceso de la juventud indígena amazónica a instancias de estudio superior en el Perú sigue siendo bastante limitado. Según el censo nacional de 1993, aproximadamente tres mil indígenas amazónicos peruanos habían accedido a, por lo menos, un año de educación superior. Este número representaría tan solo el 1,5\% del total de indígenas censados en dicho año (Espinosa, 2007b). 
En segundo lugar, la vida en las ciudades afecta el rendimiento de los jóvenes indígenas debido a los desafíos socioculturales que tienen que enfrentar. Según Roperto Noningo (2001), joven indígena awajún, los estudios superiores implican muchas veces «un fuerte desarraigo cultural» y «un choque cultural», y suelen ir acompañados, además, por «el marcado racismo en las ciudades». Todos estos elementos, según el mismo Noningo, condicionan el «desarrollo psicoemocional» de los jóvenes indígenas. La mayoría de estudiantes indígenas siente que son ignorados o discriminados al interior de sus instituciones educativas. Esta discriminación se manifiesta de diversas formas. Es frecuente que sus compañeros y a veces también los profesores se burlen de su acento o de sus costumbres, o les ponen apodos y sobrenombres ofensivos. El resultado final es una creciente falta de autoestima que les lleva muchas veces a desarrollar sentimientos de timidez, inseguridad e inferioridad, así como a la negación y el ocultamiento de su identidad indígena.

La discriminación sufrida acrecienta, además, las dificultades inherentes a la vida en un contexto distinto al propio como puede ser el ámbito urbano, lejos del medio ambiente conocido, de la familia y de los seres queridos. Los desajustes emocionales y el choque cultural que los jóvenes indígenas enfrentan en las ciudades es más acentuado en el caso de aquellos que se han desplazado fuera de la región amazónica y donde incluso el clima y la comida son radicalmente distintos. En este sentido, los estudios superiores en las propias ciudades amazónicas resultan menos traumáticas.

Eventualmente, cuando logran superar los obstáculos y desafíos de su vida en la universidad, estos jóvenes indígenas también tienen que lidiar con el desafío de ser «profesionales indígenas», es decir, personas que puedan servir de puente entre el conocimiento y la experiencia acumulada a lo largo de siglos por sus antepasados en medio de los bosques y los aportes que brindan las ciencias modernas que nacieron en Europa. Los profesionales indígenas sienten la necesidad de convertirse en los puentes que puedan facilitar la comunicación entre la tradición heredada de sus abuelos y el mundo moderno, globalizado y urbano donde viven muchos de sus amigos y contemporáneos.

Hoy en día, muchos de los líderes indígenas a nivel regional y nacional son jóvenes indígenas que han accedido a la experiencia de vivir y estudiar en una universidad o centro superior. Por ello, el futuro mismo de los pueblos indígenas dependerá en gran medida de la forma en que estos jóvenes vayan procesando la compleja experiencia de articular las tradiciones heredadas de sus antepasados con la vida urbana moderna y vayan construyendo así nuevas formas de ser indígenas amazónicos en el siglo XXI.

\section{CONCLUSIONES Y TAREAS PENDIENTES}

Los desafíos que enfrentan los indígenas amazónicos, que hoy en día viven en las ciudades, son muchos y merecen mayor atención y estudio. La información aquí presentada proviene de los resultados de una investigación preliminar que nos permite aproximarnos a la complejidad y variedad de situaciones que existen en la región amazónica peruana. Todavía queda, pues, mucho por conocer y discutir a futuro.

En primer lugar, es necesario reconocer a los indígenas urbanos como indígenas con los mismos derechos que sus paisanos que viven en las comunidades rurales. Tanto la sociedad como el Estado peruano tienen que romper con el llamado «mito del mestizaje»12 que tiende a invisibilizar y a perpetuar la discriminación contra los indígenas.

12 El término «mito del mestizaje» lo tomo prestado de Jeffrey Gould (1998). Para el caso del Perú y de los indígenas amazónicos, he elaborado a profundidad este tema en un artículo previo (Espinosa, 2003). 
En segundo lugar, hacen falta políticas públicas y acciones desde el Estado que atiendan las necesidades particulares de los indígenas urbanos. Estas políticas deberían estar orientadas no solo a mejorar sus condiciones de vida, sino también a desterrar la discriminación y el racismo que sufren los indígenas en las ciudades.

Evidentemente, para la implementación de estas acciones se requiere contar con censos y estadísticas que hoy no existen. Pero también implica abrir espacios de diálogo y debate en el que se involucren los líderes, autoridades y organizaciones indígenas. Hasta la fecha, las organizaciones indígenas, al igual que el Estado, han centrado su atención prioritaria en los indígenas que viven en las comunidades rurales, descuidando su atención a aquellos paisanos que decidieron migrar a las ciudades. Esta es también una tarea pendiente para las organizaciones indígenas.

Finalmente, la vida y los desafíos de los indígenas amazónicos que viven en las ciudades también han sido descuidados por la antropología y las ciencias sociales. Es, pues, un deber y una tarea continuar conociendo más este sector importante y creciente de la población indígena amazónica. Los antropólogos tenemos mucho que aprender de la riqueza de estrategias y experiencias de estas personas y, al mismo tiempo, debemos tratar de aportar, en la medida de nuestras posibilidades, para crear condiciones de vida más dignas y para que los derechos y la herencia cultural de los indígenas amazónicos que viven en las ciudades sean respetados y valorados.

\section{Referencias citadas}

BARRANTES, R., 2007 - Sociedades de posguerra en el Perú: secuelas de la violencia y justicia en el pueblo asháninka. In: Realidades de posguerra en el Perú: omisiones, negaciones y sus consecuencias (Félix Reátegui Carrillo, ed.): 19-48; Lima: Instituto de Democracia y Derechos Humanos de la Pontificia Universidad Católica del Perú (IDEHPUCP).

CARVAJAL, Fray G. de, 2002 [1542] - Relación del Nuevo Descubrimiento del famoso Río Grande del Amazonas. In: La Aventura del Amazonas (G. Carvajal, P. Almesto \& A. Rojas, eds.): 31-88; Madrid: Dastin.

CHAUMEIL, J.-P., 1978 - Los mellizos y la lupuna. Amazonía Peruana, 2 (3): 159-184.

COMISIÓN DE LA VERDAD Y RECONCILIACIÓN, 2003 - Los pueblos indígenas y el caso de los Asháninkas. In: Informe Final, tomo V, capítulo 2, subcapítulo 8: 241-277; Lima: Comisión de la Verdad y Reconciliación (CVR).

ESPINOSA, O., 1994 - Los Asháninka: Guerreros en una Historia de Violencia. América Indígena, 53 (4): 45-60.

ESPINOSA, O., 1998 - Los pueblos indígenas de la Amazonía Peruana y el uso político de los medios de comunicación. América Latina Hoy: Revista de Ciencias Sociales, 19: 91-100.

ESPINOSA, O., 2003 - Desafíos a la ciudadanía multicultural en el Perú: el «mito del mestizaje» y la «cuestión indígena». In: Ciudadanías inconclusas: El ejercicio de los derechos en sociedades asimétricas (N. Vigil \& R. Zariquiey, eds.): 77-89; Lima: Pontificia Universidad Católica del Perú. 
¿Cómo se relacionan con lo urbano los indígenas amazónicos peruanos en el siglo XXI?

ESPINOSA, O., 2004 - Indigenous Politics in the Peruvian Amazon: An Anthropological and Historical Approach to Shipibo Political Organization, 559 pp.; New York: New School for Social Research. Ph.D. Dissertation.

ESPINOSA, O., 2007a - Los pueblos indígenas de la Amazonía peruana ante los desafíos de la globalización. In: Apertura a la globalización: Desafíos y oportunidades en el Perú (Bernardo Haour, ed.): 15-36; Lima: Universidad Antonio Ruíz de Montoya.

ESPINOSA, O., 2007b - Para vivir mejor: los indígenas amazónicos y su acceso a la educación superior en el Perú; Santiago de Chile: Fundación Equitas. Disponible en Internet $<$ Isees.fundacionequitas.org/data/images_upload/isees_articulo_1b.pdf $>$.

GALVÃO, E., 1979 - Áreas Culturais Indígenas do Brasil: 1900-1959. In: Encontro de Sociedades: Índios e brancos no Brasil: 193-228; Rio de Janeiro: Paz e Terra.

GOULD, J., 1998 - To Die in this Way: Nicaraguan Indians and the Myth of Mestizaje, 18801965, 305 pp.; Durham: Duke University Press.

INSTITUTO NACIONAL DE ESTADÍSTICA E INFORMÁTICA (INEI), 2008 - Censos Nacionales 2007: XI de Población y VI de Vivienda. Resultados definitivos de Comunidades Indígenas, tomo 1, 621 pp.; Lima: INEI.

JARAMILLO, R., 2003 - Indiens Urbains : Processus de reconformation de l'identité ethnique indienne à Manaus, 448 pp. Tesis para obtener el grado de Doctor en Antropología en la EHESS de París.

LÉVI-STRAUSS, C., 1973 - Tristes trópicos, 419 pp.; Buenos Aires: Eudeba.

MORA, C., 1995 - Una revisión del concepto de cholo en la amazonía peruana. Amazonía Peruana, 12 (25): 145-158.

MYERS, T., 1974 - Spanish contacts and social change on the Ucayali river, Peru. Ethnohistory, 21 (2): 135-157.

NONINGO SESÉN, R., 2001 - Juventud Indígena. Voz Indígena, 1 (1).

PEIRANO, M., 2008 - Antropología sin culpa: Una visión desde Brasil. In: Saberes periféricos. Ensayos sobre la antropología en América Latina (Carlos Iván Degregori \& Pablo Sandoval, eds.): 227-247; Lima: Instituto de Estudios Peuanos, Instituto Francés de Estudios Andinos.

RIBEIRO, D., 1973 - Fronteras indígenas de la civilización, 419 pp.; México: Siglo XXI.

RIBEIRO, D., 1986 - Os índios e a civilização, 508 pp.; Petrópolis: Vozes.

SALINAS LOYOLA, J., 1965 [1557] - Descubrimientos, conquistas y poblaciones de Juan de Salinas Loyola. In: Relaciones Geográficas de Indias (Marcos Jiménez de la Espada, ed.), vol. III: 195-232; Madrid: Atlas. Biblioteca de Autores Españoles.

SANTOS GRANERO, F., 1992 - Etnohistoria de la Alta Amazonía. Siglos XV-XVIII, 305 pp.; Quito: Abya Yala, Movimiento Laico para América Latina (MLAL).

TOURNON, J., 2002 - La Merma mágica. Vida e Historia de los Shipibo-Conibo del Ucayali, 450 pp.; Lima: Centro Amazónico de Antropología y Aplicación Práctica (CAAAP).

VILLAPOLO, L., 2003 - Senderos del desengaño. Construcción de memorias, identidades colectivas y proyectos de futuro en una comunidad Asháninka. In: Jamás tan cerca arremetió lo lejos: Memoria y violencia política en el Perú (Carlos I. Degregori, ed.): 145-173; Lima: Instituto de Estudios Peruanos, Social Science Research Council.

VILLAPOLO, L. \& VÁSQUEZ, N., 1999 - Entre la guerra y el juego. Recursos psicológicos y socioculturales de los niños asháninka ante la violencia política, 155 pp.; Lima: Centro Amazónico de Antropología y Aplicación Práctica (CAAAP).

VIVEIROS DE CASTRO, E., 2004 - Perspectivismo y multinaturalismo en la América indígena. In: Tierra Adentro: Territorio indígena y percepción del entorno (Alexandre Surrallés \& Pedro García Hierro, eds.): 37-80; Lima: International Work Group for Indigenous Affairs. 